\title{
Transient Dynamics of Atoms Inside Submicron Rectangular Waveguides
}

\author{
S. Al-AWFI* \\ Department of Physics, Taibah University, P.O. Box 30002, Madina, Saudi Arabia
}

(Received March 17, 2012)

\begin{abstract}
The time evolution of the radiation pressure forces due to the action of a laser light on two-level atom moving inside a long hollow cylinder with a rectangular cross-section of sub-wavelength dimensions $a \times b$ is presented. This evolution is considered when the frequency of the light is comparable to a dipole allowed transition frequency. In this limit, the decay emission $\Gamma$ is possible only via a very small number of modes. From the solutions of the Bloch equations in the dynamic regime, we find that the transient regime, applicable from the instant the laser is switched on. This is important for the gross motion, provided that the upper-state lifetime $\Gamma^{-1}$ is relatively long while the steady-state regime, formally such that $t \gg \Gamma^{-1}$, is appropriate for the evaluation of the forces and the dynamics for large $\Gamma$. Significant variations of the characteristics of the system are emphasized. These features are illustrated using typical parameters for the case of $\mathrm{Eu}^{3+}$ that has a particularly small $\Gamma$.
\end{abstract}

DOI: $10.12693 /$ APhysPolA.123.631

PACS: 03.75.Be, 37.30.+i

\section{Introduction}

In the absence of any external effects, a dipole emitter such as neutral molecules, atoms, and ions interacts with the vacuum fields that are bound by the cavity in which the emitter is positioned. This interaction leads to alteration of the decay emission rate of the emitter to become a position-dependent. Since the pioneering work of Purcell [1] much research has been carried out, especially consequences for emitters immersed in various media and cavity structures of different shapes and sizes [2-8].

On the other hand, in the presence of any excitation cavity modes, the state of the motion of the emitter can be changed. With one of the modes that are excited with sufficient intensity, the emitter suffers a dissipative force, which is exploited in cooling (and heating) atomic motion [9]. The atom also suffers a gradient force, which is used for trapping process [9-12]. Recently, there have been a number of proposals for optical confinement, where the emitters may be trapped in two or three dimensions by a light field [13]. There are some arguments that such confinement may be useful in the study of the Bose-Einstein condensates [14]. In addition, the subject of manipulated matter in the form of neutral molecules, atoms, and ions in cavities have received an impetus with the realization that such a system can be used in quantum information processing [15-17].

However, a direct atom channel through a hollow cylindrical waveguide has been considered [12]. An evanescent mode pattern of guiding process a long hollow cylindrical waveguide has been suggested and the success was achieved in controlling atomic motion inside the submicron dimensions regime $[6,8]$. Normally the mechanisms

*e-mail: alawfi99@hotmail.com of the atomic motion in light are controlled by the optical Bloch equations and they are solved in the steady state [2-12], to illustrate the mean optical forces for a two- and three-level atom. In this case, the range of the validity of these solutions is assumed to be in the same order of the characteristic time required by the internal state of the atom to reach its steady state. It therefore seems appropriate to seek for an enlargement of the range of investigations in order to include more general types of problems, which are frequently met in practice.

The purpose of this article is to report a theory of the dynamical aspects of the Bloch equations. We consider a two-level atom in a particular cavity, namely a hollow infinite cylinder with a rectangular cross-section of sub-wavelength dimensions. We concentrate here on this type of a waveguide because of a two-level atom dynamics process based on the steady-state solutions of the optical Bloch equations inside cylindrical waveguides is fully investigated. But this process based on the exact analytic solutions for the optical Bloch equations in these structures have not been reported before. The range of the validity of the steady-state solutions is assumed to be in the same order of the characteristic time required by the internal state of the atom to reach its steady state [18-20]. Certainly, those atoms have a short lifetime of the excited state such as sodium and are not appropriate preference for considering the transient optical effects. Because the forces will rapidly converge to the steady-state case, this makes the transient forces negligible. To point out the transient effects we need to consider the transitions with a long upper-state lifetime, and rare-earth ions such as $\mathrm{Eu}^{3+}$ will be good examples.

The outline of the paper is as follows: in Sect. 2 we outline the theoretical framework beginning with a statement of the Hamiltonian appropriate for our model involving an atom of finite mass, with the internal dynamics represented by two quantum levels coupled by inter- 
action with the electromagnetic fields. We then outline the steps and justify the approximations leading to drive the optical forces that based on the steady-state solutions of the optical Bloch equations. Section 3 is devoted to the derivation of the different parameters which are related to the two-level atom, interacting with the vacuum fields that are now confined within a hollow cylindrical waveguide with rectangular cross-section. In Sect. 4, we outline the steps leading to the explicitly time dependent solutions of the optical Bloch equations. In Sect. 5, the emphasis is given to the case of the atomic motion in this structure. The essential features of the forces have been reported and discussed for the $\mathrm{Eu}^{3+}$ ion. Section 6 contains our comments and conclusions.

\section{Steady state optical forces}

We are concerned here with the evolution of the average forces acting on a two-level atom, due to its interaction with light. We assume that the light is in the form of a coherent beam with complex amplitude $\alpha$ and has a plane wave distribution. The Hamiltonian of the system reads,

$$
H=H_{\mathrm{A}}+H_{\mathrm{F}}+H_{\mathrm{int}},
$$

where $H_{\mathrm{A}}$ and $H_{\mathrm{F}}$ are the unperturbed Hamiltonian for the atom and the field, respectively, and can be written as

$$
\begin{aligned}
& H_{\mathrm{A}}=\frac{\boldsymbol{P}^{2}}{2 M}+\hbar \omega_{0} \pi^{\dagger} \pi, \\
& H_{\mathrm{F}}=\hbar \omega a^{\dagger} a,
\end{aligned}
$$

where $\boldsymbol{P}$ is the center-of-mass momentum operator, $M$ is the mass of the atom, $\omega_{0}$ is the atomic transition frequency and $\pi$ and $\pi^{\dagger}$ are the atomic raising and lowering operators. In Eq. (3), $a$ and $a^{\dagger}$ are the creation and annihilation operators for a photon with energy $\hbar \omega$. The interaction Hamiltonian $H_{\text {int }}$ in Eq. (2) describes the coupling of the atom to the electromagnetic field and is given in the electric dipole approximation at this point; it is convenient to deal with the field associated with an excited mode in the classical limit. In the rotating wave approximation, the interaction Hamiltonian may then be written as

$$
H_{\text {int }}=-\boldsymbol{\mu} \cdot \boldsymbol{E}(\boldsymbol{r}) \text {. }
$$

At this point, it is convenient to deal with the field associated with an excited mode in the classical limit. In the rotating wave approximation, the interaction Hamiltonian may then be written as

$$
H_{\text {int }}=-\mathrm{i} \hbar\left(\tilde{\pi}^{\dagger} \alpha f(\boldsymbol{r})-\text { H.c. }\right) \text {, }
$$

where $\tilde{\pi}=\pi \exp (\mathrm{i} \omega t), f(\boldsymbol{r})=\Omega(\boldsymbol{r}) \exp (\mathrm{i} \theta(\boldsymbol{r}))$ and the quantity $\Omega(\boldsymbol{r})$ is the Rabi frequency which represents the rate of the induced transition from the upper level to the lower and vice versa. It is given by

$$
\hbar \Omega(\boldsymbol{r})=\left|\alpha\langle\boldsymbol{\mu}\rangle_{12} \cdot \boldsymbol{F}\right|,
$$

where $\boldsymbol{F}$ is the mode vector function. The forces acting on the atomic center-of-mass can be derived using the optical Bloch equations for the atomic density matrix elements once we make the semi-classical approximation which allows the gross motion of the atom to be treated classically, while maintaining a quantum treatment for the internal dynamics of the atom. Thus the position and momentum operators of the center of mass may be replaced by their expectation values $\boldsymbol{r}_{0}$ and $\boldsymbol{P}_{0}$. The density matrix associated with the internal motion of the atom $\rho(t)$ evolves with time according to the well-known relation

$$
\frac{\mathrm{d} \rho}{\mathrm{d} t}=-\frac{\mathrm{i}}{\hbar}[H, \rho]+\Re \rho,
$$

where $\Re \rho$ accounts for the relaxation dynamics of the atomic system. In the adiabatic approximation, the atomic velocity $\boldsymbol{v}=\boldsymbol{P}_{0} / M$ is assumed to be constant during the time taken for the dipole moment to reach its steady-state value. The position $\boldsymbol{r}_{0}$ of the atom at time $t$ is then given by

$$
\boldsymbol{r}_{0}=\boldsymbol{r}+\boldsymbol{v} t
$$

where we have redefined $\boldsymbol{r}$ so that it now denotes the (updated) initial position of the atom. Thus we can write

$$
f\left(r_{0}\right)=f(\boldsymbol{r}+\boldsymbol{v} t) \approx f(\boldsymbol{r}) \exp (\mathrm{i}(v \nabla \theta(r) / t)),
$$

where we have assumed that the change in the field amplitude is negligible during this period of the run. Substituting the expression of $H$ in the master equation and using the aforementioned properties of $H_{\mathrm{A}}$ and $H_{\text {int }}$, we obtain the following optical Bloch equations for the atomic density matrix elements:

$$
\begin{aligned}
& \frac{\mathrm{d} \tilde{\rho}_{11}}{\mathrm{~d} t}=2 \Gamma \rho_{22}+f(\boldsymbol{r}) \tilde{\rho}_{12}+f^{*}(\boldsymbol{r}) \tilde{\rho}_{21}, \\
& \frac{\mathrm{d} \tilde{\rho}_{12}}{\mathrm{~d} t}=-[\Gamma+\mathrm{i} \Delta(\boldsymbol{r}, \boldsymbol{v})] \tilde{\rho}_{12}+f^{*}(\boldsymbol{r})\left[\rho_{22}-\rho_{11}\right], \\
& \frac{\mathrm{d} \tilde{\rho}_{21}}{\mathrm{~d} t}=-[\Gamma-\mathrm{i} \Delta(\boldsymbol{r}, \boldsymbol{v})] \tilde{\rho}_{21}+f(\boldsymbol{r})\left[\rho_{11}-\rho_{22}\right],
\end{aligned}
$$

where we use $\Gamma$ for the upper-to-lower-level decay rate, $\Delta(\boldsymbol{r}, \boldsymbol{v})=\Delta_{0}-\boldsymbol{v} \cdot \nabla \theta(\boldsymbol{r})$ is the total detuning, $\Delta_{0}=$ $\omega-\omega_{0}$ is the detuning of the field frequency from atomic resonance, $\tilde{\rho}_{21}=\langle\tilde{\pi}\rangle$ and $\tilde{\rho}_{12}=\tilde{\rho}_{21} \exp (-t v \nabla \theta(\boldsymbol{r}))$. Since the two-levels are the only ones in the problem, the conservation of probability gives

$$
\rho_{11}+\rho_{22}=1 \text {. }
$$

The mean optical force acting on the atom is defined as the average rate of change of the atomic momentum which yields

$$
\langle\boldsymbol{F}\rangle=-\left\langle\nabla H_{\text {int }}\right\rangle \text {. }
$$

After some simple algebra, the force reads

$$
\langle\boldsymbol{F}\rangle=\left\langle\boldsymbol{F}_{\text {dip }}\right\rangle+\left\langle\boldsymbol{F}_{\text {diss }}\right\rangle \text {. }
$$

This causes two types of forces to appear, a dipole force, proportional to the Rabi frequency gradient and to the in-phase component of the dipole

$$
\left\langle\boldsymbol{F}_{\text {dip }}\right\rangle=\mathrm{i} \hbar\left[\tilde{\rho}_{12}(t) f(\boldsymbol{r})-\tilde{\rho}_{21}(t) f^{*}(\boldsymbol{r})\right](\nabla \Omega(\boldsymbol{r}) / \Omega(\boldsymbol{r}))
$$

and a dissipative force, proportional to the phase gradient and to the quadrature component of the dipole

$$
\left\langle\boldsymbol{F}_{\text {diss }}\right\rangle=-\hbar\left(\tilde{\rho}_{12}(t) f(\boldsymbol{r})-\tilde{\rho}_{21}(t) f^{*}(\boldsymbol{r})\right) \nabla \theta(\boldsymbol{r}) .
$$

For given initial conditions the solution of the optical 
Bloch Eqs. (10), (11) and (12) leads formally to the determination of the forces by direct substitution in Eqs. (16) and (17). The steady state takes place when all time derivatives in the optical Bloch equations are set equal to zero and corresponds to the long-time limit. The steady-state solution of the optical Bloch equations can be written as

$$
\begin{gathered}
\left\langle\boldsymbol{F}_{\mathrm{diss}}(\boldsymbol{r}, \boldsymbol{v})\right\rangle=2 \hbar \Gamma(\boldsymbol{r}) \Omega^{2}(\boldsymbol{r}) \\
\times\left(\frac{\nabla \theta(\boldsymbol{r})}{\Delta(\boldsymbol{r}, \boldsymbol{v})+2 \Omega^{2}(\boldsymbol{r})+\Gamma(\boldsymbol{r})}\right), \\
\left\langle\boldsymbol{F}_{\mathrm{dip}}(\boldsymbol{r}, \boldsymbol{v})\right\rangle=-2 \hbar \Omega(\boldsymbol{r}) \Delta(\boldsymbol{r}, \boldsymbol{v}) \\
\times\left(\frac{\nabla \Omega(\boldsymbol{r})}{\Delta(\boldsymbol{r}, \boldsymbol{v})+2 \Omega^{2}(\boldsymbol{r})+\Gamma(\boldsymbol{r})}\right) .
\end{gathered}
$$

In our recent paper $[18,19]$ we have recognized that there were some special cases of the interest where the original optical Bloch equations can be solved analytically. Then the transient effects can be incorporated using our treatment. We shall report and discuss the results of these phenomena in the last section.

\section{Confined optical structure}

We consider a two-level atom, interacting with the vacuum fields that are now confined within optical structure in form of hollow cylindrical waveguides with a circular cross-section, as illustrated in Fig. 1. As shown in this figure, a normal cross-section is assumed to have the dimensions $a \times b$ and is taken to lie in the $y-z$ plane with the cylinder axis along the $x$-direction, coinciding with the straight line $y=b / 2, z=a / 2$. The cylindrical waveguide is bounded by walls arising from the intersection of four planes at $y=0, y=b$ and $z=0, z=a$, all are assumed to be planar surfaces of perfect conductors which exclude all electromagnetic fields from their interior. Once again, the standard electromagnetic boundary conditions apply such that the tangential component of the electric field vector and the magnetic field vector must vanish at every point on all the guide walls.

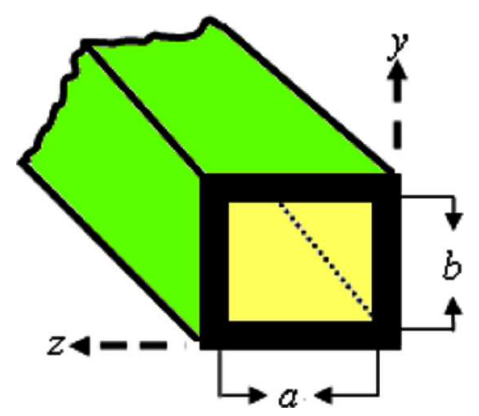

Fig. 1. Schematic drawing of the hollow rectangular waveguide.

It is well-known that such confined optical structure leads to two types of physical effects. First, the decay emission rate of the atom is modified and, second, the atom experiences energy shifts to both levels. In this paper we are just concerned with the first one, for the details. Using Fermi's golden rule with this symmetric structure, the decay emission rate $\Gamma$ is independent of the coordinate $x$ (i.e. parallel axis) and can be evaluated for the three possible orientations for a dipole situated at an arbitrary point $(0, b / 2, a / 2)$ as follows [21]:

$$
\begin{gathered}
\Gamma_{\|}(y, z)=\Gamma_{0} \sum_{m=0}^{[2 b / \lambda]} \sum_{n=0}^{[2 a / \lambda]} \frac{3 \lambda^{3}}{4 \pi^{2} a^{2} b} G_{n m}^{x}, \\
\Gamma_{\perp}(y, z)=\Gamma_{0} \sum_{m=0}^{[2 b / \lambda]} \sum_{n=0}^{[2 a / \lambda]}\left(\frac{3 \lambda}{b}\right) \\
\times\left(\frac{1}{f_{m n}} F_{n m}^{y, z}+\frac{\lambda^{2}}{4 \pi^{2} a^{2}} G_{n m}^{y, z}\right),
\end{gathered}
$$

where $G$ and $F$ functions appearing in above equation are given by

$$
\begin{aligned}
& G_{m n}^{x}=\frac{\pi^{2}\left(a^{2} m^{2} / b^{2}+n^{2}\right) \sin ^{2}(m \pi y / b) \sin ^{2}(n \pi z / a)}{\left[\left(\omega_{0} a / c\right)^{2}-m^{2} \pi^{2} a^{2} / b^{2}-n^{2} \pi^{2}\right]^{1 / 2}} \\
& G_{m n}^{y}= \\
& \frac{m^{2} a^{2}\left(a^{2} m^{2} / b^{2}+n^{2}\right)^{-1} \cos ^{2}(m \pi y / b) \sin ^{2}(n \pi z / a)}{b^{2}\left[\left(\omega_{0} a / c\right)^{2}-m^{2} \pi^{2} a^{2} / b^{2}-n^{2} \pi^{2}\right]^{-1 / 2}} \\
& G_{m n}^{z}= \\
& \frac{n^{2}\left(a^{2} m^{2} / b^{2}+n^{2}\right)^{-1} \sin ^{2}(m \pi y / b) \cos ^{2}(n \pi z / a)}{\left[\left(\omega_{0} a / c\right)^{2}-m^{2} \pi^{2} a^{2} / b^{2}-n^{2} \pi^{2}\right]^{-1 / 2}} \\
& F_{m n}^{y}= \\
& \frac{n^{2}\left(a^{2} m^{2} / b^{2}+n^{2}\right)^{-1} \cos ^{2}(m \pi y / b) \sin ^{2}(n \pi z / a)}{\left[\left(\omega_{0} a / c\right)^{2}-m^{2} \pi^{2} a^{2} / b^{2}-n^{2} \pi^{2}\right]^{1 / 2}} \\
& F_{m n}^{z}= \\
& \frac{m^{2} a^{2}\left(a^{2} m^{2} / b^{2}+n^{2}\right)^{-1} \sin ^{2}(m \pi y / b) \cos ^{2}(n \pi z / a)}{b^{2}\left[\left(\omega_{0} a / c\right)^{2}-m^{2} \pi^{2} a^{2} / b^{2}-n^{2} \pi^{2}\right]^{1 / 2}}
\end{aligned}
$$

where $\Gamma_{0}$ is the free-space decay rate, $\lambda$ is the free space transition wavelength and $n, m$ refer to the order of excited mode while $[2 b / \lambda]$ and $[2 a / \lambda]$ stands for the integer part of the bracketed quantity. All expressions of the decay emission rate can easy be plotted as distribution in three dimensions as functions of the ratio $y / b$ and $z / a$.

On the other hand, the position-dependent Rabi frequency $\Omega$, which characterizes the interaction of an atom with the electric fields and for $\mathrm{TM}_{11}$ excited mode, $\Omega$ for three possible orientations is given by [21]:

$$
\begin{aligned}
& \Omega_{x}^{11}(y, z) \\
& =\Omega_{0}\left(\frac{2 c}{\omega_{0}}\right)\left(\frac{\pi^{2}}{b^{2}}+\frac{\pi^{2}}{a^{2}}\right) \sin \left(\frac{\pi y}{b}\right) \sin \left(\frac{\pi z}{a}\right), \\
& \Omega_{y}^{11}(y, z)=\Omega_{0}\left(\frac{2 c k \pi}{b \omega_{0}}\right)\left(\frac{\pi^{2}}{b^{2}}+\frac{\pi^{2}}{a^{2}}\right)^{-1 / 2} \\
& \quad \times \cos \left(\frac{\pi y}{b}\right) \sin \left(\frac{\pi z}{a}\right),
\end{aligned}
$$




$$
\begin{aligned}
& \Omega_{z}^{11}(y, z)=\Omega_{0}\left(\frac{2 c k \pi}{a \omega_{0}}\right)\left(\frac{\pi^{2}}{b^{2}}+\frac{\pi^{2}}{a^{2}}\right)^{-1 / 2} \\
& \times \sin \left(\frac{\pi y}{b}\right) \cos \left(\frac{\pi z}{a}\right),
\end{aligned}
$$

where $\Omega_{0}$ is the free-space Rabi frequency and $k$ is an axial wave vector inside the rectangular waveguide which is given by

$$
k=\left[(\omega / c)^{2}-(m \pi / b)^{2}-(n \pi / a)^{2}\right]^{1 / 2} .
$$

The last factor which must be considered in this context is the laser light detuning. For such an optical system the dynamic detuning is given by the following expression [21]:

$$
\Delta(y, z, n, m, \boldsymbol{v})=\Delta_{0}-(\boldsymbol{v} \cdot k \hat{\boldsymbol{x}}),
$$

where the static detuning $\Delta_{0}=\omega_{0}-\omega(k, n, m)$.

These different factors will be introduced in the original optical Bloch equations which can be solved by using the treatment developed in $[18,19]$, we outline here the essential steps for the solution.

\section{Dynamical aspects in the optical Bloch equation}

It is possible to make the atom density matrix equations of the motion (8)-(10) resemble those for a magnetic dipole undergoing precession in a magnetic field. This approach has value not only in solving the equations, but also in providing a physical picture of the density matrix in motion. The equations we derive here are equivalent to the Bloch equations treated in [20]. On the other hand, for several useful situations, a single level decay constant is a good approximation so that the Bloch model is accurate and may be easier to use. The solutions obtained are used in the discussion of light forces in the considered structure.

We introduce the real quantities

$$
\begin{aligned}
& U=\hat{\rho}_{21}+\text { c.c., } \\
& V=\mathrm{i} \hat{\rho}_{21}+\text { c.c. } \\
& W=\rho_{22}-\rho_{11},
\end{aligned}
$$

in terms of which

$$
\hat{\rho}_{21}=(1 / 2)(U-\mathrm{i} V) \text {. }
$$

These quantities are very little in an optical period and are the components of the vector Bloch $\boldsymbol{B}$. Taking derivatives of Eqs. (32), (33) and (34) and using (12), (13), we find the Bloch equations

$$
\begin{aligned}
& \dot{U}=-\Gamma U+\Delta(\boldsymbol{r}, \boldsymbol{v})+2 \Re(f(\boldsymbol{r})) W, \\
& \dot{V}=-\Gamma V-\Delta(\boldsymbol{r}, \boldsymbol{v}) U-2 \wp(f(\boldsymbol{r})) W, \\
& \dot{W}=-2 \Gamma(W+1)-2 \Re f(\boldsymbol{r}) U+2 \wp(f(\boldsymbol{r})) V .
\end{aligned}
$$

Here $\dot{W}$ equation is written for the case of upper to lower level decay. This system can be written in matrix form as

$$
\left(\begin{array}{c}
\dot{U} \\
\dot{V} \\
\dot{W}
\end{array}\right)=\left(\begin{array}{ccc}
-\Gamma & \Delta & 2 \Re(f) \\
-\Delta & -\Gamma & -2 \wp(f) \\
-2 \Re(f) & 2 \wp(f) & -2 \Gamma
\end{array}\right)\left(\begin{array}{c}
U \\
V \\
W
\end{array}\right)
$$

$$
+\left(\begin{array}{c}
0 \\
0 \\
-2 \Gamma
\end{array}\right) .
$$

These equations are similar to those of Bloch studied in [20]. The same treatment can be used here to obtain the solutions of this linear system. This system can be written in the matrix form as

$$
\dot{\boldsymbol{B}}=\Psi \boldsymbol{B}+\Lambda,
$$

where $\boldsymbol{B}$ is the Bloch vector, $\Psi$ is a non-diagonal $(3 \times 3)$ matrix and $\Lambda$ is an inhomogeneous vector. The general solution of the linear coupled system (39) is

$$
\boldsymbol{B}=\boldsymbol{B}_{\mathrm{h}}+\boldsymbol{B}_{\mathrm{p}},
$$

where $\boldsymbol{B}_{\mathrm{h}}$ is the homogeneous solution of the system without the term $\Lambda$ and $\boldsymbol{B}_{\mathrm{p}}$ is the particular solution. By choosing $\dot{\boldsymbol{B}}=0$ in the system (39), the particular solution can be obtained $\boldsymbol{B}_{\mathrm{p}}=\Psi^{-1} \Lambda$ which is the steady-state solution of given Eqs. (8) and (9).

Consider now the homogeneous system

$$
\boldsymbol{B}_{\mathrm{h}}=\Psi \boldsymbol{B} \text {. }
$$

The eigenvalues of the homogeneous system satisfy the characteristic equation

$$
\begin{aligned}
& (\Gamma+\lambda)^{2}(2 \Gamma+\lambda)+4|f(\boldsymbol{r})|^{2}(\Gamma+\lambda) \\
& \quad+[\Delta(\boldsymbol{r}, \boldsymbol{v})]^{2}(2 \Gamma+\lambda)=0,
\end{aligned}
$$

where $|f(\boldsymbol{r})|^{2}=|\Omega(\boldsymbol{r})|^{2}$ is the Rabi frequency, as we have seen that the Rabi frequency depends only on the normal coordinate $y, z$, then the solutions depend on the same component. In fact, the eigenvalues give us all the information we need to know about how the homogeneous solution behaves in time. It is possible that two of the eigenvalues can be complex numbers that if $\lambda_{2}=\lambda_{3}^{*}=a \pm \mathrm{i} b$ then the homogeneous solutions can be rearranged so that they are of the form

$$
\begin{aligned}
& h(t)=A_{h 1} \exp \left(\lambda_{1} t\right)+A_{h 2} \exp (a t) \cos (b t) \\
& \quad+A_{h 3} \exp (a t) \sin (b t),
\end{aligned}
$$

where $h(t)$ stands for either $U, V$, or $W$, and the $A^{\prime}$ s coefficients are constants to be determined from the initial conditions.

\section{Applications}

Here we emphasize the submicron cavity dimensions in the rectangular waveguides context. Our aim is to describe the optical forces that act on the ion, the manner in which such forces change the state of motion in the steady state and the transient optical regime. The state of the motion of the ion inside the rectangular waveguide can be altered by the excitation of one or more cavity modes. With one of the modes excited with sufficient intensity, the ion experiences radiation forces of the type familiar in the case of ions subject to spatially varying light in free space [20]. On the other hands, the solutions of the optical Bloch equations show that the dipole moment and hence the forces acting on the ion relax to steady state values outside a time of the order $5 \Gamma^{-1}$ from 
the instant at which the light field is switched on [18-20]. Since this time is typically much greater than the observation times involved in the channeling of ions through the structure, we need to investigate the effects of the transient state forces.

To show the efficacy of our treatment and the effects of the transient regimes, we shall focus on the dissipative forces. The explicit form of the dissipative force is given in Eq. (17) which contains the factor $\nabla \theta$ corresponding to the momentum imparted by light to the ion. However, in view of equations of the electric field inside this optical structure [21], the mode phase for all modes is given by $\theta=k x$. Therefore, the gradient of the mode phase is $\nabla \theta=k \hat{\boldsymbol{x}}$. This immediately fixes the direction of the dissipative force as parallel to the longitudinal axis of the waveguide

$$
\left\langle\boldsymbol{F}_{\text {diss }}\right\rangle=-\hbar\left[\tilde{\rho}_{12}(t) f(r)-\tilde{\rho}_{21}(t) f^{*}(r)\right] k .
$$

To study the transient effects we need to consider the transitions with a long upper state lifetime, and rare-earth ions are good examples of this ultimate. In particular, we consider $\mathrm{Eu}^{3+}$ ion in the calculations with the following parameters [18]: $\lambda=614 \mathrm{~nm}, \Gamma_{0}=$ $1.1 \times 10^{3} \mathrm{~s}^{-1}, \Omega_{0}=3.9 \times 10^{7} \mathrm{~s}^{-1}$ and $k=1.0 \times 10^{7} \mathrm{~m}^{-1}$, and in the case, $\Delta(r, v)=0$ with the initial condition $v(0)=0$. In Figs. 2 and 3 we display the dissipative force in terms of time at the point $x=0$ with different positions of the ion on the normal cross-section within rectangular waveguide for the case, when the $p$-polarization cavity mode is excited and the electric dipole matrix is oriented parallel to the waveguide. The oscillatory behavior persists again and the time of the transient effects is about three times of the decay rate.

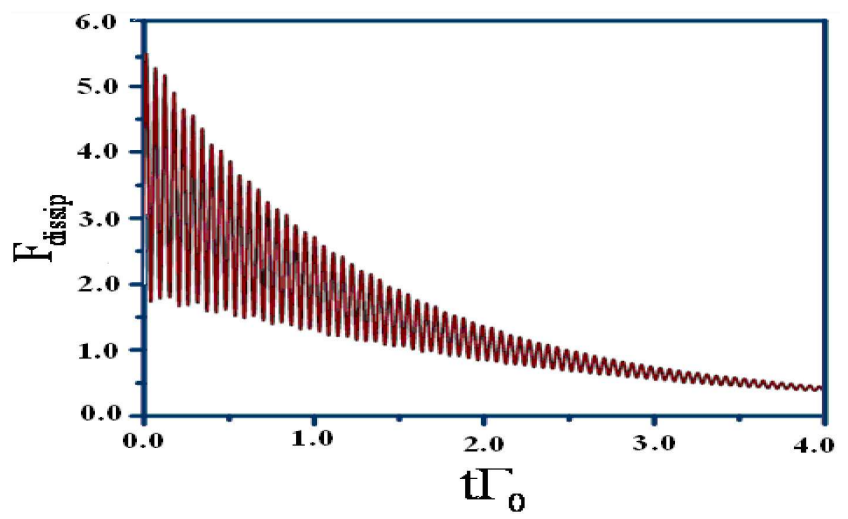

Fig. 2. Dynamic dissipative force (in unit of $\hbar k \Omega$ ) when the atom fixed at the point $(x=0, y=b / 2$, $z=a / 2)$ as a function of time, when $\mathrm{TM}_{11}$ mode is excited and dipole matrix is oriented parallel to guide axis.

Figure 4 shows the dynamic dissipative force acting on $\mathrm{Eu}^{3+}$ when the $p$-polarization cavity mode is excited and the electric dipole moment matrix is oriented normal to the waveguide for the point $x=0$ with different positions of the ion on the normal cross-section within

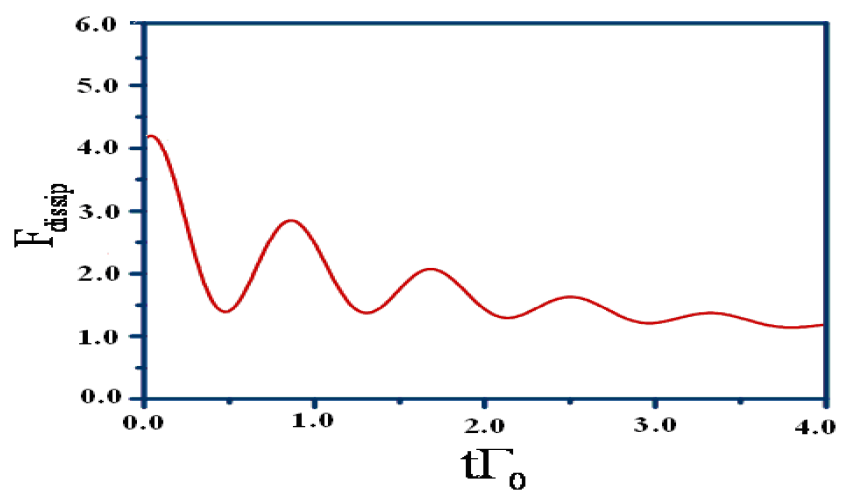

Fig. 3. Dynamic dissipative force (in unit of $\hbar k \Omega$ ) when the atom fixed at the point $(x=0, z=a / 2)$ with different position on the $y$ axis, as a function of time, when $\mathrm{TM}_{11}$ mode is excited and dipole matrix is oriented parallel to guide axis.

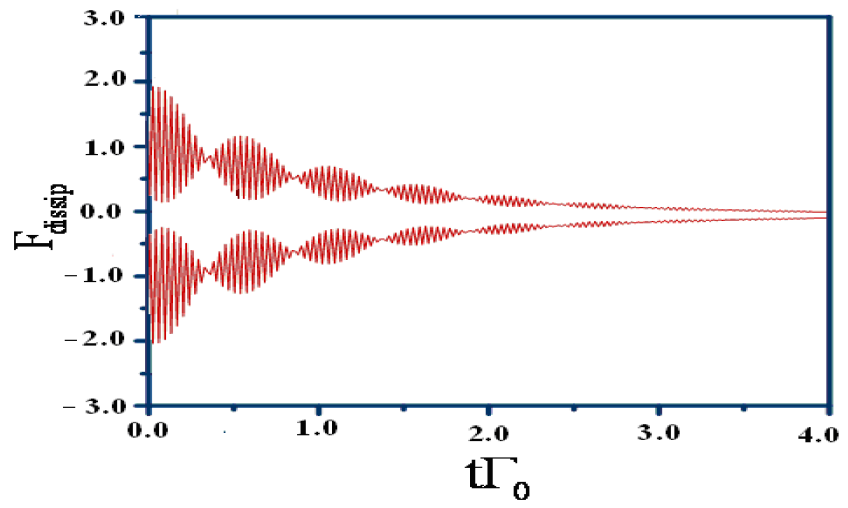

Fig. 4. Dynamic dissipative force (in unit of $\hbar k \Omega$ ) when the atom fixed at the point $(x=0, z=a / 2)$ with different position on the $y$ axis, as a function of time, when $\mathrm{TM}_{11}$ mode is excited and dipole matrix is oriented normal to the guide axis.

rectangular waveguide. The remarkable point in these cases is the decay of the force in an oscillatory manner, with manifestation of the beats phenomena. On the other hand, we mention here that the dissipative force inside the waveguide is evaluated at some positions. If the atom moves then it acquires velocity, which means its position changes with time and the dynamics detuning delta also changes with the velocity. So, the forces are evaluated at fixed positions as time passes, but the atom is not there, as it has moved on. This important point may be examined in detail by solving the classical motion of the center of mass of the atom.

\section{Conclusion}

We have focused here only on cylindrical atom waveguides characterized by two distinct features. First the guide is assumed to have a rectangular cross-section and second, the walls of the waveguide are taken to be perfect 
conductors. As obvious, lines of extension of this work should consider cylindrical perfect conductor atom guides with circular cross-sections also of subwavelength dimensions. Although the atomic motion in hollow submicron circular cylinders based on the steady-state optical forces has been completely evaluated, the effects of the dynamical aspects optical forces along the lines discussed in this paper for these structures have not been reported before. It is well known that the electromagnetic modes of circular cylindrical guides have azimuthal components which give more significance of the transient dynamics effects on the helical motion of atoms.

A related problem that could be studied is that of cylindrical atom guides with guide walls made of dielectrics characterized by dispersive dielectric functions which could also exhibit loss. Although the decay emission of atoms in dielectric waveguides has been investigated [8], the atomic motion neither with steady state nor dynamical aspects optical forces in this structure have been reported before. Work along these lines is now in progress and the results will be reported in due course.

\section{References}

[1] E. Purcell, Phys. Rev. 69, 681 (1946).

[2] M. Ol'Shanii, Y. Ovchinnkov, V. Letokhov, Opt. Commun. 98, 77 (1993).

[3] S. Marksteiner, C. Savage, P. Zoller, S. Rolston, Phys. Rev. A 50, 2680 (1994).

[4] M. Rippin, P. Knight, J. Mod. Opt. 47, 807 (1996).

[5] S. Al-Awfi, M. Babiker, Phys. Rev. A 58, 2274 (1998).

[6] J. Dowling, J. Gea-Banacloche, Adv. Atom. Mol. 37, 1 (1996).
[7] S. Al-Awfi, Int. J. Mod. Phys. B 18, 515 (2004).

[8] G. Banyard, C. Bennett, M. Babiker, Opt. Commun. 207, 195 (2002).

[9] C. Adams, E. Riss, Prog. Quant. Electron. 21, 1 (1997).

[10] M. Babiker, W. Power, L. Allen, Phys. Rev. Lett. 73, 1239 (1994).

[11] W. Power, L. Allen, M. Babiker, V. Lembessis, Phys. Rev. A 52, 479 (1995).

[12] V. Paranjape, P. Panat, S. Lawande, Phys. Rev. A 64, 063817 (2001).

[13] D. Boiron, C. Triche, D. Meacher, P. Verkerk, G. Grynberg, Phys. Rev. A 52, R3425 (1995).

[14] J.P. Dowling, J. Gea-Banacloche, Phys. Rev. A 52, 3997 (1995).

[15] J. Ye, D. Vernooy, H. Kimble, Phys. Rev. Lett. 83, 4987 (1999).

[16] A. Doherty, T. Lynn, C. Hood, H. Kimble, Phys. Rev. A 63, 013401 (2000).

[17] A. Mundt, A. Kreuter, C. Becher, D. Leibfried, J. Eschner, F. Schmidt-Kaler, R. Blatt, Phys. Rev. Lett. 89, 103001 (2002).

[18] S. Bougouffa, S. Al-Awfi, J. Mod. Opt. 55, 473 (2008).

[19] S. Bougouffa, S. Al-Awfi, Appl. Math. Inform. Sci. 3, 355 (2009).

[20] A. Carter, M. Babiker, M. Al-Amri, D. Andrews, Phys. Rev. A 72, 043307 (2005).

[21] S. Al-Awfi, M. Babiker, Phys. Rev. A 58, 4768 (1998). 